\title{
A NOTE ON MINIMAL ENVELOPES OF DOUGLAS ALGEBRAS, MINIMAL SUPPORT SETS, AND RESTRICTED DOUGLAS ALGEBRAS
}

\author{
CARROLL GUILLORY
}

(Received 9 December 2000)

\begin{abstract}
We characterize the interpolating Blaschke products of finite type in terms of their support sets. We also give a sufficient condition on the restricted Douglas algebra of a support set that is invariant under the Bourgain map, and its minimal envelope is singly generated.
\end{abstract}

2000 Mathematics Subject Classification. 46J15, 46J30.

1. Introduction. Let $H^{\infty}$ be the Banach algebra of bounded analytic functions on the open unit disk $D$. We denote by $M\left(H^{\infty}\right)$ the set of nonzero complex valued homomorphism of $H^{\infty}$. With the weak*-topology, $M\left(H^{\infty}\right)$ is a compact Hausdorff space. We identify a function in $H^{\infty}$ with the Gelfand transform and consider $H^{\infty}$ the supremum norm closed subalgebra of the space of continuous functions on $M\left(H^{\infty}\right)$. By Carleson's corona theorem, $D$ is dense in $M\left(H^{\infty}\right)$ in the weak ${ }^{*}$-topology. For $f \in H^{\infty}$, put

$$
\begin{gathered}
Z(f)=\left\{x \in M\left(H^{\infty}\right) \backslash D: f(x)=0\right\}, \\
\{|f|<1\}=\left\{x \in M\left(H^{\infty}\right) \backslash D:|f(x)|<1\right\} .
\end{gathered}
$$

For two points $x, y$ in $M\left(H^{\infty}\right)$, the pseudohyperbolic distance is given by

$$
\rho(x, y)=\sup \left\{|f(y)|: f \in H^{\infty},\|f\|_{\infty} \leq 1, f(x)=0\right\} .
$$

Then, $0 \leq \rho(x, y) \leq 1$ and put

$$
P(x)=\left\{m \in M\left(H^{\infty}\right): \rho(x, m)<1\right\} .
$$

The set $P(x)$ is called the Gleason part containing $x$. For $z, x \in D, \rho(z, w)=$ $|(z-w) /(1-\bar{w} z)|$, and $P(z)=D$. When $P(x) \neq\{x\}$, both $x$ and $P(x)$ are called nontrivial. We denote by $G$ the set of nontrivial points in $M\left(H^{\infty}\right)$.

For an infinite sequence $\left\{z_{n}\right\}_{n}$ in $D$ with $\sum_{n=1}^{\infty}\left(1-\left|z_{n}\right|\right)<\infty$, the corresponding Blaschke product is defined by

$$
b(z)=\prod_{n=1}^{\infty} \frac{-\bar{z}_{n}}{\left|z_{n}\right|} \frac{z-z_{n}}{1-\bar{z}_{n} z}, \quad z \in D .
$$

In addition, we have

$$
\inf _{n}\left(1-\left|z_{n}\right|^{2}\right)\left|b^{\prime}\left(z_{n}\right)\right|>0
$$


both $b$ and $\left\{z_{n}\right\}_{n}$ are called interpolating. When $b$ is interpolating and

$$
\lim _{n \rightarrow \infty}\left(1-\left|z_{n}\right|^{2}\right)\left|b^{\prime}\left(z_{n}\right)\right|=1,
$$

both $b$ and $\left\{z_{n}\right\}_{n}$ are called sparse. An interpolating Blaschke product $b$ is said to be unimodular on trivial points if $\{x:|b(x)|<1\} \subset G$. In [4], Hoffman proved that for $x \in M\left(H^{\infty}\right), x \in G$ if and only if $x \in Z(b)$ for some interpolating Blaschke product $b$. He also proved that for a point $x \in G$, there exists a one-to-one continuous onto map $L_{x}: D \rightarrow P(x)$ such that $L_{x}(0)=x$ and $f \circ L_{x} \in H^{\infty}$ for every $f \in H^{\infty}$. The map $L_{x}$, which is called the Hoffman map for the point $x$, is given by

$$
L_{x}(z)=\lim _{\alpha} \frac{z+z_{0}}{1+\bar{z}_{\alpha} z}, \quad z \in D,
$$

where $\left\{Z_{\alpha}\right\}_{\alpha}$ is a net in $D$ which converges to $x$. A part $P(x)$ is called sparse if there is a sparse Blaschke product $b$ such that $b(x)=0$. In this case we have $\left|\left(b \circ L_{x}\right)^{\prime}(0)\right|=1$. Therefore, $b$ is a sparse Blaschke product if and only if $\left|\left(b \circ L_{x}\right)^{\prime}(0)\right|=1$ for every $x \in Z(b)$. A part is called locally sparse if there is an interpolating Blaschke product $b$ such that $b(x)=0$ and $\left|\left(b \circ L_{x}\right)^{\prime}(0)\right|=1$.

For an interpolating Blaschke product $b$ with zeros $\left\{z_{n}\right\}_{n}$, let

$$
\delta_{0}(b)=\lim _{n \rightarrow \infty} \inf \min _{k \neq n} \rho\left(z_{n}, z_{k}\right) .
$$

An interpolating Blaschke product $b$ is called spreading if $\delta_{0}(b)=1$. By considering boundary function, we may consider $H^{\infty}$, as a closed subalgebra of $L_{\infty}$, the Banach algebra of essentially bounded Lebesgue measurable functions on the unit circle $T$. It is known that $M\left(L^{\infty}\right) \subset M\left(H^{\infty}\right)$ and $M\left(L^{\infty}\right)$ is the Shilov boundary for $H^{\infty}$. Any uniformly closed subalgebra $B$ with $H^{\infty} \subset B \subset L^{\infty}$ is called a Douglas algebra. For a point $x \in M\left(H^{\infty}\right)$, there exists a probability measure $\mu_{x}$ on $M\left(L^{\infty}\right)$ such that

$$
f(x)=\int_{M\left(L^{\infty}\right)} f d \mu_{x} \quad \forall f \in H^{\infty} .
$$

We denote by supp $\mu_{x}$ the closed support set of $\mu_{x}$. Since supp $\mu_{x}$ is a weak peak set of $M\left(L^{\infty}\right)$ for $H^{\infty}$, we have $H_{\text {supp } \mu_{x}}^{\infty}=\left\{f \in L^{\infty}: f_{\mid \operatorname{supp} \mu_{x}} \in H_{\mid \text {supp } \mu_{x}}^{\infty}\right\}$ is a Douglas algebra.

For $E \subset M\left(H^{\infty}\right)$, a point $x \in E$ is called a minimal support point for $E$ if

$$
\operatorname{supp} \mu_{x} \subset \operatorname{supp} \mu_{y} \quad \text { or } \quad \operatorname{supp} \mu_{x} \cap \operatorname{supp} \mu_{y}=\phi \quad \forall y \in E \text {. }
$$

If $x$ is a minimal support point for $E, \operatorname{supp} \mu_{x}$ is called a minimal support set for $E$. For an interpolating Blaschke product $b$, we denote by $m(Z(b))$ the set of minimal support points for the set $\{x:|b(x)|<1\}$. Let $X$ be a Banach algebra with identity and let $B$ be a closed subalgebra of $X$. The Bourgain algebra $B_{b}$ of $B$ relative to $X$ is defined by the set of $f$ in $X$ such that $\left\|f f_{n}+B\right\| \rightarrow 0$ for every sequence $\left\{f_{n}\right\}_{n}$ in $B$ with $f_{n} \rightarrow 0$ weakly. If $A$ and $B$ are Douglas algebras with $A \subseteq B$ and properly contained, then $B$ is a minimal superalgebra of $A$ if and only if $\operatorname{supp} \mu_{x}=\operatorname{supp} \mu_{y}$ for every $x, y \in M(A) \backslash M(B)$. We denote by $B_{m}$ the smallest Douglas algebra which contains all minimal superalgebras of $B$. We note that $B_{b} \subset B_{m}$. An interpolating Blaschke product $b$ such that $\{x:|b(x)|<1\} \subset G$, with $Z(b) \cap P(x)$ being a finite set for every $x \in Z(b)$, is said to be of finite type. 


\section{Proofs of the theorems}

THEOREM 2.1. An interpolating Blaschke product $b$ that is unimodular on trivial parts is of finite type if and only if $m(Z(b))=\{z:|b(z)|<1\}$.

Proof. Suppose $b$ is an interpolating Blaschke product that is unimodular on the trivial points and of finite type. Let $z \in M\left(H^{\infty}+C\right)$ such that $|b(z)|<1$. By [1, Theorems 1 and 2], there is an $x \in m(Z(b))$ such that supp $\mu_{x} \subset \operatorname{supp} \mu_{z}$. By [3, Theorem 3.1], the set $\operatorname{supp} \mu_{x}$ is a maximal support set. Hence $\operatorname{supp} \mu_{x}=\operatorname{supp} \mu_{z}$. This implies that $z$ is a minimal support point for $b$, that is, $z \in m(Z(b))$. So $\{z$ : $|b(z)|<1\} \subset m(Z(b))$. Since $m(Z(b)) \subset\{z:|b(z)|<1\}$, we have $\{z:|b(z)|<1\}=$ $m(Z(b))$. Conversely, suppose $m(Z(b))=\{z:|b(z)|<1\}$ and assume that $b$ is unimodular on trivial points but not of finite type. Then there is a $y \in Z(b)$ such that the set $Z(b) \cap P(y)$ is an infinite set. By [2, Theorems 1 and 2], there is an $x \in M\left(H^{\infty}+C\right)$ such that $|b(x)|<1$, an uncountable index set $I$ such that for $\alpha, \beta \in I$, $\alpha \neq \beta, \operatorname{supp} \mu_{x_{\alpha}} \cap \operatorname{supp} \mu_{x_{\beta}}=\phi, x_{\alpha}, x_{\beta} \in m(Z(b))$, and $\operatorname{supp} \mu_{x_{\alpha}} \subset \operatorname{supp} \mu_{x}$ for all $\alpha \in I$. Since supp $\mu_{x_{\alpha}}$ is properly contained in supp $\mu_{x}$, this implies that $x \notin m(Z(b))$ but $|b(x)|<1$. This contradicts our assumption that $\{z:|b(z)|<1\}=m(Z(b))$. Thus, $b$ is of finite type.

THEOREM 2.2. Suppose that $b$ is a spreading nonsparse Blaschke product, and $x \in$ $m(Z(b))$ such that $\left|\left(b \circ L_{x}\right)^{\prime}(0)\right| \neq 1$. Then

(i) $\left(H_{\operatorname{supp} \mu_{x}}^{\infty}\right)_{b}=H_{\operatorname{supp} \mu_{x}}$,

(ii) $\left(H_{\operatorname{supp} \mu_{x}}^{\infty}\right)_{m}=H_{\operatorname{supp} \mu_{x}}^{\infty}[\bar{b}]$.

Proof. By [5, Lemma 2.1], we have that $P_{x}$ is a nonlocally sparse part. Hence, by [6, Theorem 5] we have that (i) holds.

Since $b$ is spreading and $x \in m(Z(b))$,

$$
M\left(H_{\operatorname{supp} \mu_{x}}^{\infty}\right)=M\left(H_{\text {supp } \mu_{x}}^{\infty}[\bar{b}]\right) \cup E_{x},
$$

where $E_{x}=\left\{y \in M\left(H^{\infty}+C\right): \operatorname{supp} \mu_{x}=\operatorname{supp} \mu_{y}\right\}$. This implies that $H_{\operatorname{supp} \mu_{x}}^{\infty}$ is properly contained in $\left(H_{\mathrm{supp} \mu_{x}}^{\infty}\right)_{m}$. Since $H_{\mathrm{supp} \mu_{x}}^{\infty}$ is a maximal subalgebra of $H_{\mathrm{supp} \mu_{x}}^{\infty}[\bar{b}]$, $H_{\text {supp } \mu_{x}}^{\infty}[\bar{b}]$ is contained in $\left(H_{\text {supp } \mu_{x}}^{\infty}\right)_{m}$. Since

$$
M\left(H_{\operatorname{supp} \mu_{x}}^{\infty}\right)=M\left(L^{\infty}\right) \cup\left\{y \in M\left(H^{\infty}+C\right): \operatorname{supp} \mu_{y} \subseteq \operatorname{supp} \mu_{x}\right\},
$$

we show that if $q$ is an interpolating Blaschke product such that $\bar{q} \in\left(H_{\operatorname{supp} \mu_{x}}^{\infty}\right)_{m}$, then $H_{\text {supp } \mu_{x}}^{\infty}[\bar{q}]=H_{\text {supp } \mu_{x}}^{\infty}[\bar{b}]$. This proves (ii). Suppose that we have $H_{\text {supp } \mu_{x}}^{\infty}[\bar{b}]$ properly contained

in $\left(H_{\text {supp } \mu_{x}}^{\infty}\right)_{m}$, then we have $M\left(\left(H_{\operatorname{supp} \mu_{x}}^{\infty}\right)_{m}\right)$ properly contained in $M\left(H_{\operatorname{supp} \mu_{x}}^{\infty}[\bar{b}]\right)$. So there is a $y \in M\left(H_{\text {supp } \mu_{x}}^{\infty}[\bar{b}]\right)$, an interpolating Blaschke product $q$ with $\bar{q} \in\left(H_{\text {supp } \mu_{x}}^{\infty}\right)_{m}$ and $q(y)=0$. By (2.2) we have $y \in M\left(H_{\text {supp } \mu_{x}}^{\infty}\right)$ but $y \notin E_{x}$. Again, by (2.2), this implies that supp $\mu_{y}$ is properly contained in the supp $\mu_{x}$. By [2, Theorems 1 and 2], there is an uncountable index set $I$ such that if $\alpha, \beta \in I, \alpha \neq \beta$, there are $x_{\alpha}, x_{\beta} \in Z(q)$ with $\operatorname{supp} \mu_{\alpha} \cap \operatorname{supp} \mu_{x_{\beta}}=\phi$ and $\operatorname{supp} \mu_{\alpha}$, supp $\mu_{x_{\beta}}$ are both properly contained in supp $\mu_{x}$. This implies that

$$
\cup_{\alpha \in I} E_{x_{\alpha}} \subset\left\{m \in M\left(H_{\text {supp } \mu_{x}}^{\infty}\right):|q(m)|<1\right\} .
$$


But this contradicts [2, Theorem 3] since $\alpha \neq \beta$ implies that $E_{x_{\alpha}} \cap E_{x_{\beta}}=\phi$. Thus, no such $y$ exists and we have $H_{\text {supp } \mu_{x}}^{\infty}[\bar{b}]=H_{\text {supp } \mu_{x}}^{\infty}[\bar{q}]$. So (ii) holds.

\section{REFERENCES}

[1] C. Guillory and K. Izuchi, Maximal Douglas subalgebras and minimal support points, Proc. Amer. Math. Soc. 116 (1992), no. 2, 477-481. MR 92m:46075. Zbl 760.46046.

[2] - Minimal envelopes of Douglas algebras and Bourgain algebras, Houston J. Math. 19 (1993), no. 2, 201-222. MR 94i:46067. Zbl 816.46048.

[3] _ Interpolating Blaschke products of type G, Complex Variables Theory Appl. 31 (1996), no. 1, 51-64. MR 97i:46090. Zbl 865.30054.

[4] K. Hoffman, Bounded analytic functions and Gleason parts, Ann. of Math. (2) 86 (1967), 74-111. MR 35\#5945. Zbl 192.48302.

[5] K. Izuchi, Spreading Blaschke products and homeomorphic parts, Complex Variables Theory Appl. 40 (2000), no. 4, 359-369. MR 2001c:46103.

[6] R. Mortini and R. Younis, Douglas algebras which are invariant under the Bourgain map, Arch. Math. (Basel) 59 (1992), no. 4, 371-378. MR 94c:46105. Zbl 760.46050.

CARroll Guillory: Department of MAthematics, University of Louisiana AT LAFAYETTE, LAFAYETTE, LA 70504, USA

E-mail address: cjg2476@1ouisiana.edu 


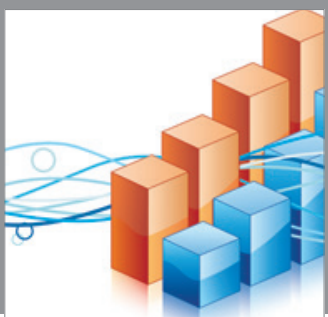

Advances in

Operations Research

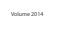

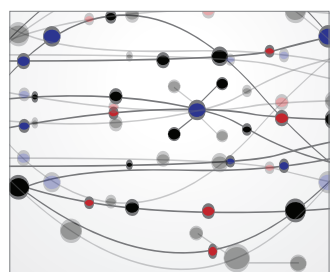

\section{The Scientific} World Journal
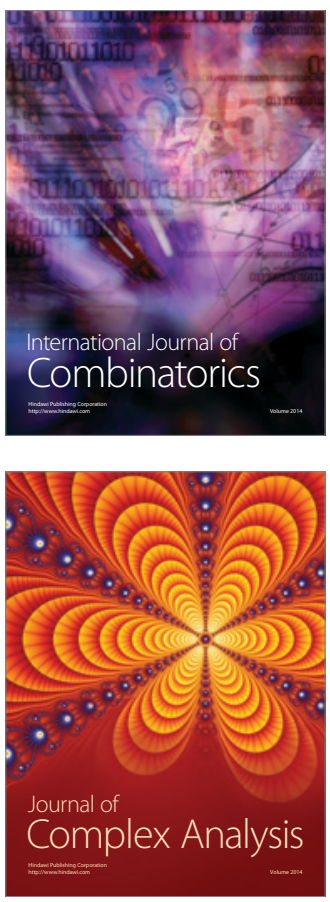

International Journal of

Mathematics and

Mathematical

Sciences
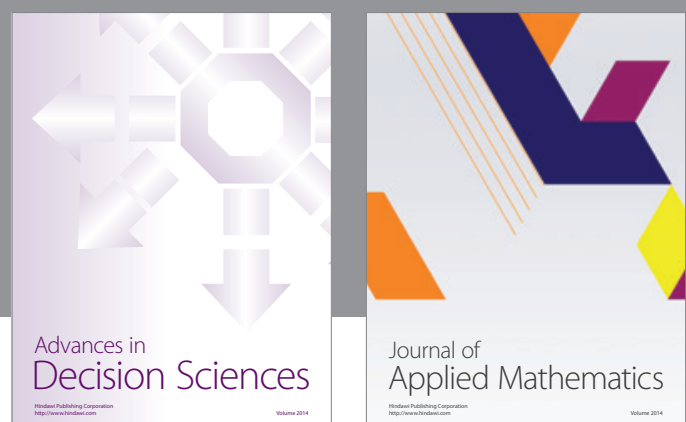

Journal of

Applied Mathematics
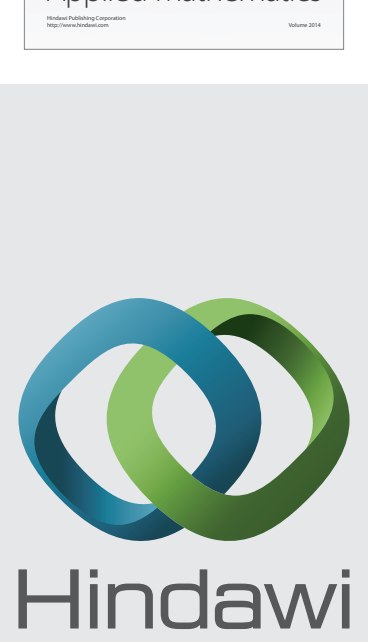

Submit your manuscripts at http://www.hindawi.com
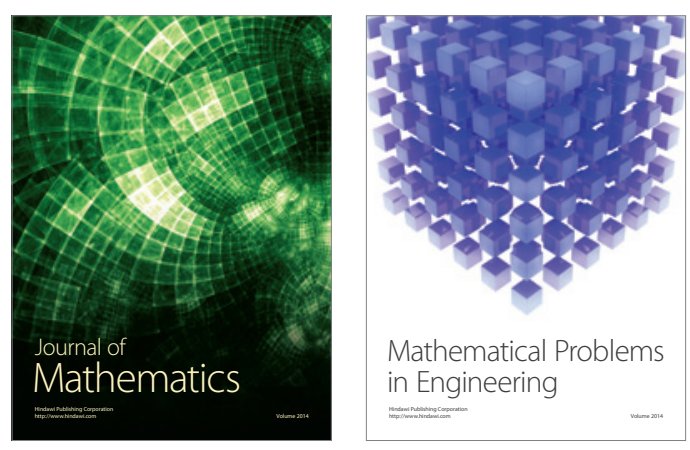

Mathematical Problems in Engineering
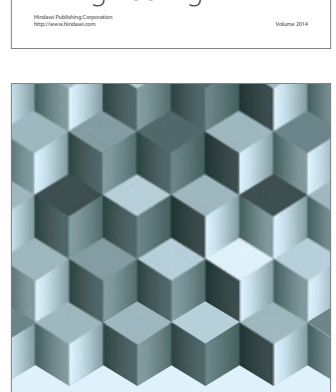

Journal of

Function Spaces
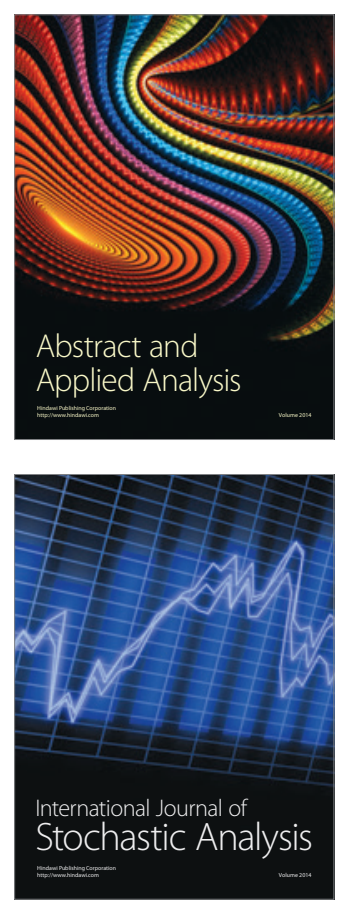

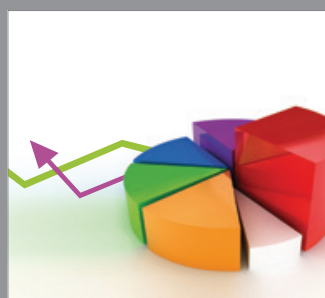

ournal of

Probability and Statistics

Promensencen
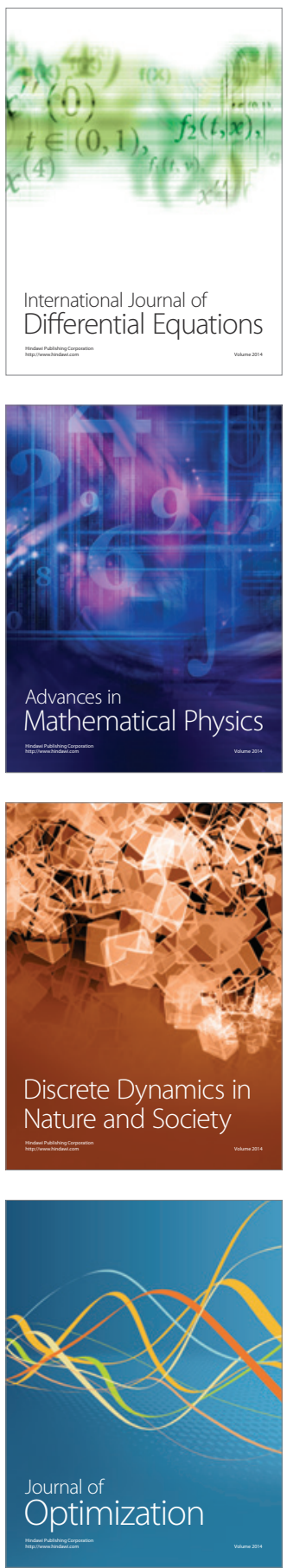